\title{
Assessment of the CSL and SU models for bcc-Fe grain boundaries
}

\section{from the first principles}

\author{
Jian $\mathrm{Xu}^{1,2}$, Yong Jiang ${ }^{1,3,4 *}$, Litong Yang ${ }^{1,2 *}$, Jiangxu $\mathrm{Li}^{5}$ \\ ${ }^{1}$ Key Laboratory of Nonferrous Materials (MOE), School of Materials Science and Engineering, Central \\ South University, Changsha, 410083, China \\ ${ }^{2}$ Key Laboratory of Advanced Materials (MOE), School of Materials Science and Engineering, Tsinghua \\ University, Beijing 100084, China \\ ${ }^{3}$ National Key Laboratory of Powder Metallurgy, Central South University, Changsha, 410083, China \\ ${ }^{4}$ Shenzhen Research Institute of Central South University, Shenzhen, 518057, China \\ ${ }^{5}$ Shenyang National Laboratory for Materials Science, Institute of Metal Research, Chinese Academy of \\ Science, Shenyang, 110016, China
}

\begin{abstract}
We present a systematic first principles investigation on a group of representative low- $\Sigma(\Sigma \leq 11)$ symmetric tilt grain boundaries in bcc-Fe. The grain boundary (GB) structures were constructed using both the coincident site lattice (CSL) and structural unit (SU) models. Calculations are performed to address the relation and applicability of the two models. Results suggest that on some of the GBs, the CSL and SU models may yield different atomistic structures. In these cases, their structures differ only by one vacancy, and the SU model always predicts a much lower GB formation energy than the CSL does. Further calculations on GB vacancy formation suggest that the SU model is more appropriate for describing the low- $\Sigma$ bcc GBs.
\end{abstract}

\section{Keywords}

Fe, grain boundary, coincident site lattice model, structural unit model, first principles

* Corresponding authors.

E-mail address: yjiang@csu.edu.cn (Y. Jiang); ltyang@csu.edu.cn (L. Yang)

(C) 2016. This manuscript version is made available under the Elsevier user license http://www.elsevier.com/open-access/userlicense/1.0/ 


\section{INTRODUCTION}

The development of nuclear energy as an environmentally clean energy source has been consistently stimulated in recent decades, for it not only reduces our reliance on fossil fuels, but also promisingly offers an eventual solution to the world's energy crisis. The viability of nuclear energy ultimately depends on the development of high performance structural materials that can support extended component lifetime under extremely hostile conditions in fission and fusion reactors [1]. Ferritic alloys are among the most promising candidates for such applications [2]. During service, high energy neutrons $(E>1 \mathrm{Mev})$ produced from $(\mathrm{n}, \alpha)$ transmutation reactions generate an ultra-high number of insoluble helium atoms, vacancies, and self-interstitial atoms in the ferrite matrix [3]. The reliability and lifetime of ferritic alloy components in nuclear applications suffer seriously from the segregation and enrichment of these defects at grain boundaries (GBs), leading to the severe loss of toughness at lower irradiation temperatures, and degradation of creep rupture properties at higher temperatures [4]

Grain boundary defines the interfacial region between two adjacent grains, with a crystallographic structure distinct from the grain interiors. Since the concept of "grain boundary design" was proposed by Watanabe [5], GBs have attracted increasing research attention in recent years. By acting as sinks and sources of point defects, GBs can play critical roles in atom diffusion and dislocation motion in metallic matrix, and thus greatly influence grain growth, recrystallization, creep, superplasticity, as well as the radiation damage effects [5-7]. Historically perhaps due to the experimental difficulties in GB characterization, numerous theoretical efforts have been made to model GB structures in proper manners, and further to assess the relevant energetics and properties. The employed models are rather diverse, being mostly the coincident-site-lattice (CSL) model $[8,9]$ or the structural-unit (SU) model $[10,11]$, and in fewer cases, the dislocation model $[7,12]$, the disclination model $[13,14]$, the O-lattice model $[15,16]$, or the displacement-shift-complete (DSC) model [17]. In particular, the CSL model is constructed by rotating one grain lattice against the other about a rotation axis by a certain angle, so as to achieve a number of coincident lattice sites [9]. The degree of fit $(\Sigma)$ between the two adjoining lattices is described by the reciprocal of the ratio of coincidence sites to the total number of sites. Thus a lower $\Sigma$ boundary corresponds to a boundary plane that contains a higher density of coincident sites. Deviations from the ideal CSL orientation may be accommodated by local atomic relaxation or the inclusion of dislocations into the boundary. While in the SU 
model, those grain boundaries with certain misorientation angles correspond to "favored" structural units (typically with a low $\Sigma$ value), and each structural unit can be seen as a favored symmetric tilt grain boundary (STGB). All other boundaries with long periods can be characterized as a combination of structural units comprising one or several neighboring favored boundaries with shorter periods [18].

It is surprising to know from the literatures that, the predicted formation energy of a given GB can be very different when using different GB models, even by the same research group. For some of the $<110>$ and $<100>$ tilt Fe-GBs, the CSL and SU models can predict almost the same formation energy by first principles calculations [19-26], but for the others, the CSL predicted formation energies are always 30 50\% higher than the SU counterparts [22, 23, 27]. To our best knowledge, there has never been a first-principles study aimed to evaluate the relation between the two mainstream models. To calculate a given GB, it is still not clear which model shall be more appropriate to use. Moreover, no first-principles study has been performed based on the same one model that thus allows a direct comparison between the two representative STGBs of bcc phase, for example, the $\langle 110\rangle$ and $\langle 100\rangle$ tilt Fe-GBs. The interaction of GBs with point defects such as vacancies is also far from being understood. The aim of the present work is to make up for these loopholes. The paper is organized as follows. The methodologies for GB modeling and calculations are described in Sec. II. In Sec. III, intensive DFT calculations are performed: (i) to predict the stable GB configurations and the corresponding formation energies for a group of low- $\Sigma(\Sigma \leq 11)$ special GBs within the $\langle 100\rangle$ and $\langle 110\rangle$ symmetric tilt grain boundary systems; (ii) to clarify the relation between the CSL and the SU models for bcc-Fe GBs; (iii) to manifest the vacancy interaction with some exemplary GBs. In Sec. IV, we summarize all our major results and findings. The obtained knowledge will be very helpful in guiding our future study on the GB-related problems in ferritic alloys for nuclear applications.

\section{CALCULATION METHODS}

To construct a GB structure using either model, the CSL or the SU model, two different approaches are often followed in the literatures. The first approach employs a supercell containing only one GB interface between two grain lattices, with a sufficient vacuum thickness to isolate the two extra, identical free surfaces at both ends [28] (see Fig. 1(a)). When evaluating the GB formation energy, the extra energy due to the two free surfaces must be dissociated from the total free energy of the supercell ensemble. Our many practices 
revealed that this approach can be problematic when the two free surfaces are highly unstable, say, with high Miller indices. Severe surface relaxation and even surface reconstruction may take place. Thus the calculated surface energy and, consequently, the GB formation energy can be very unfaithful. The second approach employs a "sandwich" supercell that contains two identical GB interfaces. The two interfaces are separated by a sufficient thickness of one grain lattice (see Fig. 1(b)), so that the vacuum and the two extra free surfaces can be avoided. The calculation accuracy can be also assured effectively, as long as a full relaxation is carefully performed both on supercell shape and volume. With this consideration, we employ the "sandwich" supercell approach for all calculations throughout this work. Furthermore, one study of Suzuki and Mishin [29] has suggested that the impact of point defects on GBs is rather short-ranged, being limited within only a few atomic layers from GBs. Thus, when constructing the sandwich supercell models, we feel comfortable to include only the nearest five to six layers from the GB interface, and regard it to be sufficient even for calculations of vacancy on GBs.

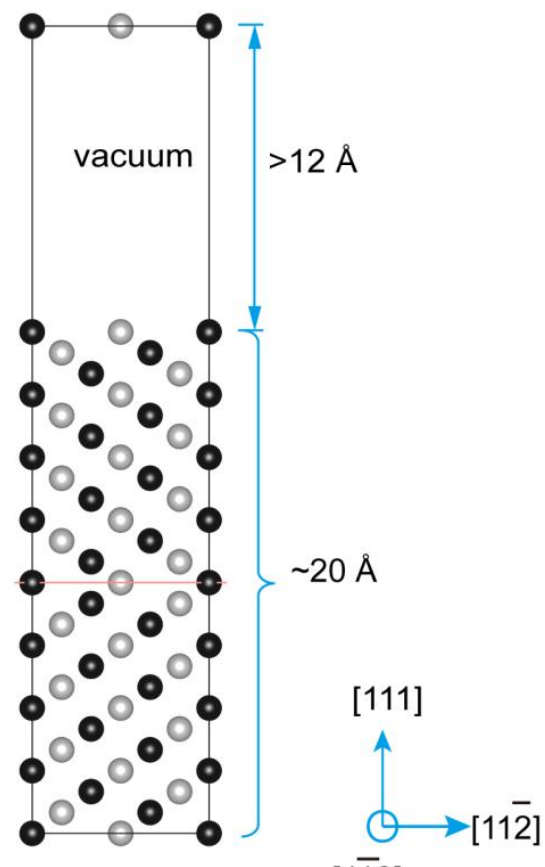

(a)

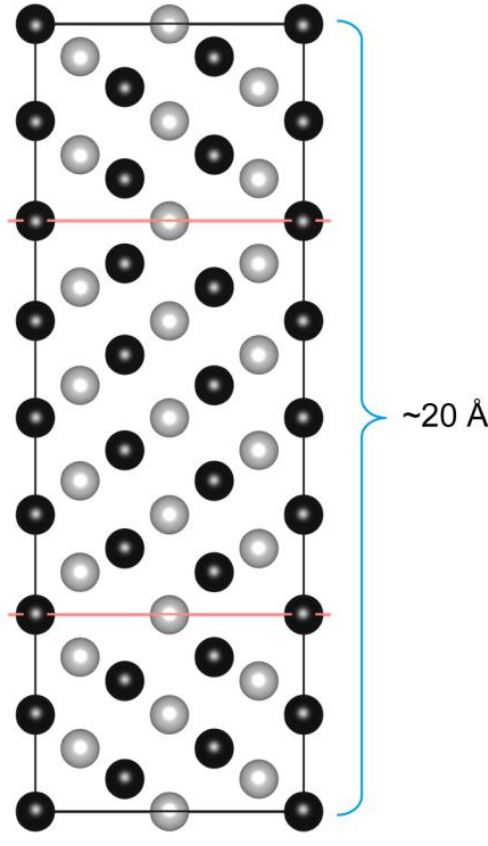

(b)

FIG.1 The GB supercells constructed using (a) the first and (b) the second approach for the $\Sigma 3(111)[1 \overline{1} 0]$ bcc-Fe STGB. The use of gray and black balls is to differentiate two adjacent (110) atom layers.

All calculations were performed in the framework of density functional theory (DFT) as implemented 
in the Vienna Ab initio Simulation Package (VASP) [30]. The electron-ion interactions were described by the projector augmented wave method (PAW) within the frozen-core approximation [31, 32]. The exchange-correlation energy was treated in the spin-polarized generalized gradient approximation (GGA) with the Perdew-Burke-Ernzerhof (PBE) functional [33]. A kinetic-energy cutoff of $350 \mathrm{eV}$ was tested to be sufficient for plane-waves expansion to achieve good convergence. Bcc $\alpha$-Fe was assumed in its ferromagnetic state. Our predictions on the equilibrium lattice constant and bulk modulus were $2.840 \AA$ and 174.7 GPa respectively, both in good agreement with the experimental values of $2.867 \AA$ [34] and $168 \mathrm{GPa}$ [35]. The magnetic moment per Fe atom was predicted as $2.17 \mu_{B}$, close to the experimental value of $2.22 \mu_{B}[35]$ and other calculations of $2.13 \sim 2.32 \mu_{B}[26,36-39]$.

The GB energy $\left(\gamma_{G B}\right)$ can be evaluated as

$$
\gamma_{G B}=\frac{1}{2 A}\left(E_{G B}-E_{b u l k}\right)
$$

where $\mathrm{A}$ is the area of the GB. $E_{G B}$ and $E_{b u l k}$ are the total energies of the sandwich GB supercell and the perfect bulk lattice with the exactly same number of atoms, respectively. The two energies were calculated with the same k-mesh density to minimize numerical errors.

\section{RESULTS AND DISCUSSION}

\section{A Configurations and energies of different GB models}

Table I summarizes our results for various low- $\Sigma$ CSL GBs $(\Sigma \leq 11)$ within the $<100>$ and $<110>$ STGB systems, as compared to all available results in literature. The comparison was made between the CSL and SU models and between DFT and molecular dynamics (MD) methods, in terms of GB configuration, misorientation angle $(\theta)$, GB model, total number of modeled atoms $(\mathrm{N})$, and GB formation energy $\left(\gamma_{G B}\right)$. Note that, no matter which model was used, MD methods always underestimated the GB formation energy. Our results agree well with all previous DFT results. Most notably, for the $\Sigma 3\{112\}<110\rangle, \Sigma 3\{111\}<110\rangle$, or $\Sigma 5\{310\}<100>$ GB, the CSL and SU models yield the same GB structure and thus the exactly same values of $\gamma_{G B}$, while for the $\Sigma 5\{210\}<100>, \Sigma 9\{114\}<110>$, or $\Sigma 11\{332\}<110>$ GB, the two models yield different structures, and the SU model always predict a much lower formation energy than the CSL does. 
Table I. Calculation results for all low- $\Sigma$ STGBs of bcc-Fe, in comparison with all literature values.

\begin{tabular}{|c|c|c|c|c|c|}
\hline Grain boundary & $\theta(\mathrm{deg})$ & model & $\mathbf{N}$ & $\gamma_{\mathrm{GB}}\left(\mathbf{J} / \mathbf{m}^{2}\right)$ & Other calculations \\
\hline \multirow{2}{*}{$\Sigma 3\{112\}<110\rangle$} & \multirow{2}{*}{70.53} & \multirow{2}{*}{$\mathrm{CSL} / \mathrm{SU}$} & 24 & 0.44 & DFT-CSL:0.44[19], 0.47[20], 0.34[21] \\
\hline & & & 48 & 0.43 & MD:0.26[40], 0.262[18] \\
\hline \multirow{3}{*}{$\Sigma 3\{111\}<110\rangle$} & \multirow{3}{*}{109.47} & \multirow{3}{*}{$\mathrm{CSL} / \mathrm{SU}$} & 48 & 1.52 & DFT-CSL:1.52[21], 1.51[22], 1.57[23], \\
\hline & & & & & $1.61[27], 1.66[41]$ \\
\hline & & & 96 & 1.53 & MD:1.308[40], 1.23[42], 1.297[18] \\
\hline \multirow{3}{*}{$\Sigma 5\{310\}<100>$} & \multirow{3}{*}{36.87} & \multirow{3}{*}{$\mathrm{CSL} / \mathrm{SU}$} & 40 & 1.57 & \multirow{3}{*}{$\begin{array}{c}\text { DFT-CSL:1.53[20], } 1.51[24], 1.48[22] \\
1.48[25], 1.63[26] \\
\text { MD:1.01[40], 0.987[18] }\end{array}$} \\
\hline & & & & & \\
\hline & & & 80 & 1.53 & \\
\hline \multirow{4}{*}{$\Sigma 5\{210\}<100>$} & \multirow{4}{*}{53.13} & CSL & 40 & 2.12 & \multirow{4}{*}{$\begin{array}{c}\text { DFT-CSL: } 2.00[23] \\
\text { MD-SU: } 1.113[40], 1.096[18]\end{array}$} \\
\hline & & SU & 38 & 1.61 & \\
\hline & & CSL & 80 & 2.11 & \\
\hline & & SU & 76 & 1.61 & \\
\hline \multirow{3}{*}{$\Sigma 9\{114\}<110\rangle$} & \multirow{3}{*}{38.94} & CSL & 72 & 2.01 & DFT-CSL: 2.09[22] \\
\hline & & $\mathrm{SU}$ & 68 & 1.38 & \multirow{2}{*}{$\begin{array}{c}\text { DFT-SU: } 1.50[22] \\
\text { MD-SU: } 1.286[40], 1.146[18]\end{array}$} \\
\hline & & SU & 136 & 1.40 & \\
\hline \multirow{2}{*}{$\Sigma 11\{332\}<110>$} & \multirow{2}{*}{50.48} & CSL & 44 & 2.17 & \multirow{2}{*}{$\begin{array}{c}\text { DFT-SU: } 1.49[27] \\
\text { MD-SU: } 1.020[40], 1.018[18]\end{array}$} \\
\hline & & SU & 42 & 1.38 & \\
\hline
\end{tabular}

The above calculations involved many details that need to be carefully handled as we will address below. For the sake of brevity, we now demonstrate our practices on two representative coincident-site-lattice (CSL) modeled GBs, the $\Sigma 5(310)$ [001] and the $55(210)$ [001] STGBs (Fig. 2). Recall that the CSL and SU models predicted the same value of formation energy on the $\Sigma 5(310)$ [001] STGB but failed on the $\Sigma 5(210)[001]$ STGB. Following the standard procedure for CSL model construction [8], the $\Sigma 5(310)[001] \mathrm{GB}$ was created by rotating one bcc-Fe grain against the other around the [001] axis by $36.87^{\circ}$. The resulting supercell in Fig. 2(a) contains 80 atoms and has a dimension of $5.68 \times 8.98 \times 17.96 \AA^{3}$. A Monkhorst-Pack [43] k-mesh of $3 \times 2 \times 1$ was employed for Brillouin zone integration. The $55(210)[001]$ GB was created by rotating one grain by about $53.13^{\circ}$. The resulting supercell in Fig. 2(b) contains 40 atoms and has a dimension of $2.84 \times 6.35 \times 25.40 \AA^{3}$. An $8 \times 4 \times 1$ Monkhorst-Pack k-mesh was employed. The separation between the two identical GB interfaces are $8.98 \AA$ and $12.70 \AA$, respectively, which are sufficient to 
eliminate the GB-GB interaction along z-axis due to the imposed periodic boundary conditions.
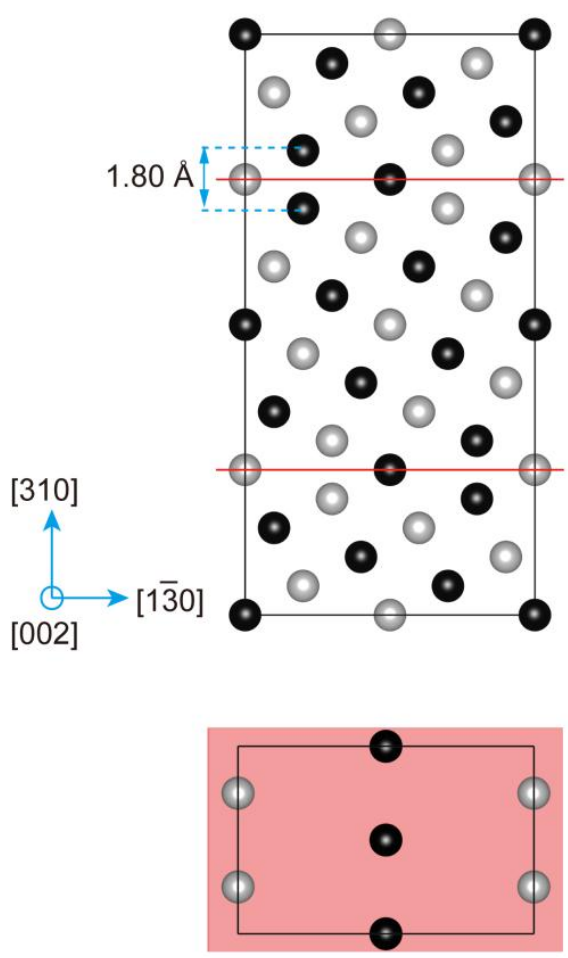

(a)

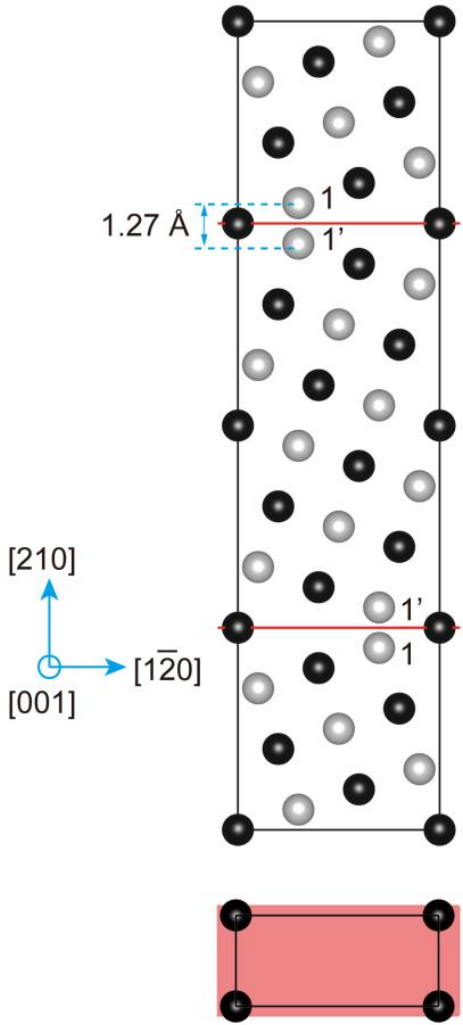

(b)

FIG. 2. The CSL model structures for (a) the $\Sigma 5(310)[001]$ and (b) the $\Sigma 5(210)[001]$ STGBs. Note each structure possesses the inversion symmetry to ensure the identity between the two embedded GB interfaces.

It must be noted here that, $\alpha$-Fe(310) has an interlayer spacing of $\sim 0.90 \AA$, and thus the CSL model structure of $\Sigma 5(310)[001] \mathrm{GB}$ is naturally reasonable for allowing for a smooth atomic relaxation. In contrast, the interlayer spacing of $\alpha$-Fe (210) is only $0.635 \AA$, leading to a too small interatomic distance of $1.27 \AA$ (labeled as the 1-1' pair in Fig. 2b) in the CSL-model structure of $\Sigma 5(210)[001]$ GB. This unreasonably small interatomic distance always caused the collapse of the GB structure during subsequent relaxation calculation. To solve this problem, two methods have been suggested in literatures [25, 28]. Method I: to artificially widen the (1-1') interatomic spacing to a more reasonable value before initiating the relaxation, say $1.78 \AA$ as shown in Fig. 3(b). In such way, full relaxation can be allowed smoothly, finally leading to an optimized CSL-GB configuration shown in Fig. 3(c). Method II: to artificially remove one atom (1 or 1') and then manually adjust the other atom to restore the mirror symmetry about the GB interface (shown in Fig. 3(d)). 
One notices that Method II essentially reproduced the SU model of $\Sigma 5(210)[001]$ GB. In the CSL model of $\Sigma 5(210)[001]$ GB, each atomic layers of (210) hosts one iron atom, as seen in Fig. 3(b) before relaxation and Fig. 3(c) after relaxation. While in the SU model of $\Sigma 5(210)[001]$ GB, the GB interface now hosts two iron atoms, and the sandwich supercell contains only 38 atoms in total, as seen in Fig. 3(d) before relaxation and Fig. 3(e) after relaxation. Comparing Figs. 3(c) and (e), it is strongly suggested that the two models for this GB differ exactly by one GB vacancy. To confirm this, a full relaxation calculation was then carried out on a vacancy-containing CSL GB as shown in Fig. 3(f). The vacancy was created by simply removing one iron from the sub-interface layer. Indeed, the optimized configuration after full relaxation exactly reproduced the fully relaxed SU model as seen in Fig. 3(e).

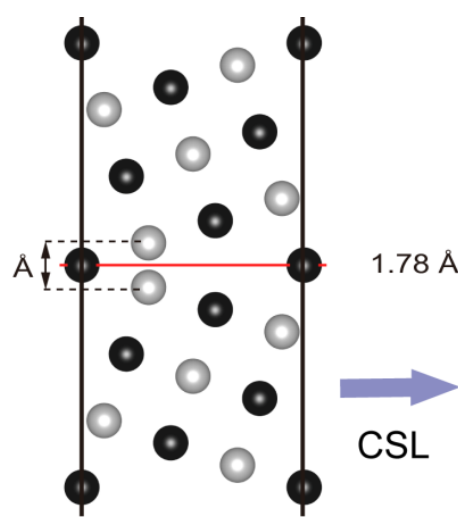

(a)

(d)

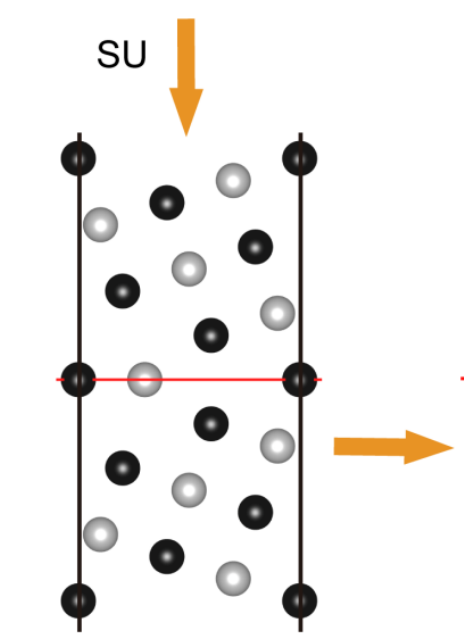

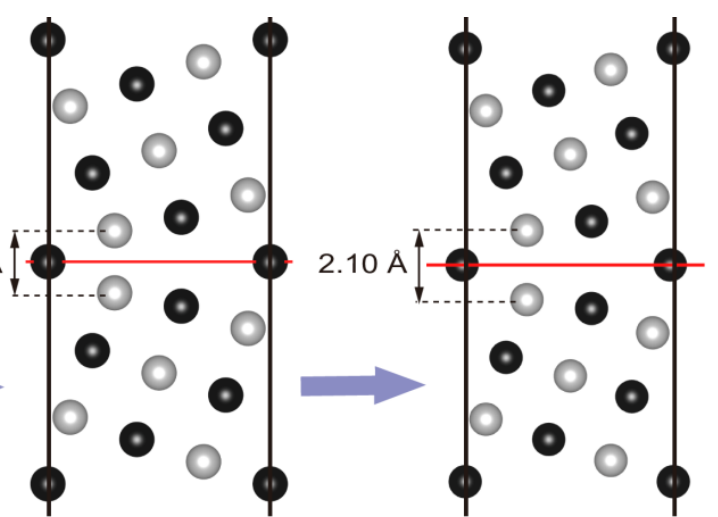

(b)

(c)

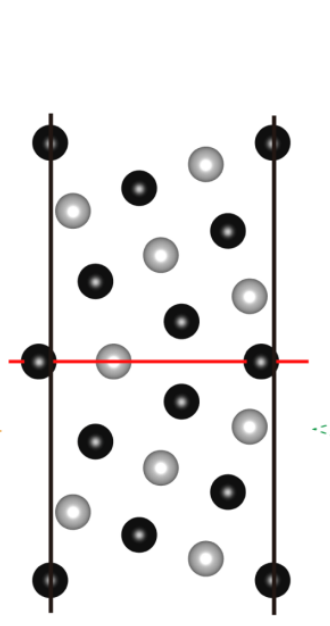

(e)

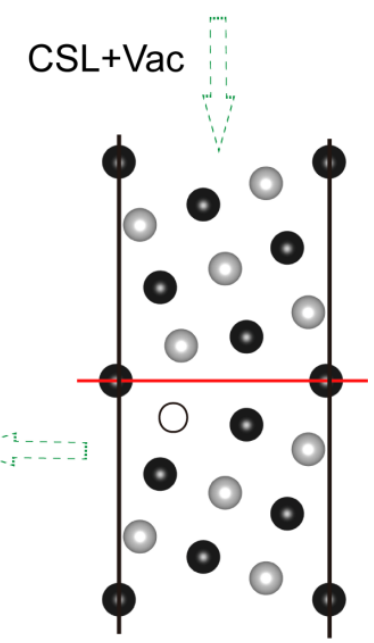

(f)

FIG. 3. Initial (a, b, d) and relaxed (c, e) schematics of atomic sites of $\Sigma 5(210)$ [001] STGB projected onto the (100) plane. Here, (b) and (c) refer to the initial and relaxed CSL model, while (d) and (e) refer to the initial and relaxed SU model, respectively. Black and gray denote atoms on different $\{100\}$ plane. The open circle in (f) corresponds to a vacancy created in the CSL model of (c). 
The SU models of all the considered low- $\Sigma \alpha$-Fe STGBs are plotted in Fig. 4. As already mentioned above, for the $\Sigma 3\{112\}<110>, \Sigma 3\{111\}<110>$, and $\Sigma 5\{310\}<100>$ STGBs, the CSL and the SU models yield exactly the same structure and thus the same GB formation energy. However, for the $\Sigma 5\{210\}<100>$, $\Sigma 9\{114\}<110\rangle$, and $\Sigma 11\{332\}<110>$ STGBs, the SU model structure is different from the CSL one, and the former predicts a much lower GB formation energy. We have also shown clearly in Fig. 3 that the CSL and the SU models for the $\Sigma 5(210)[001]$ STGB differ only by a vacancy on this GB, or in other words, the CSL-model $\Sigma 5(210)[001]$ GB can spontaneously transform into a SU configuration when introducing a GB vacancy. Thus the GB formation energies of the two models differ by one vacancy formation energy on the CSL GB. Extensive calculations were further carried out for the $\Sigma 9\{114\}<110>$ and $\Sigma 11\{332\}<110>$ STGBs, where the same relation between the CSL and SU model were confirmed.
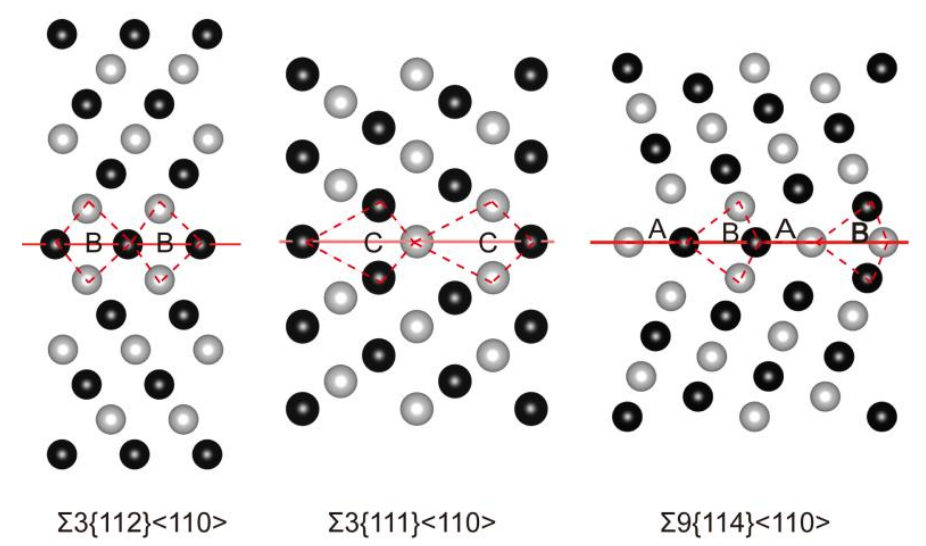

$$
\Sigma 3\{111\}<110>
$$

$\sum 9\{114\}<110>$
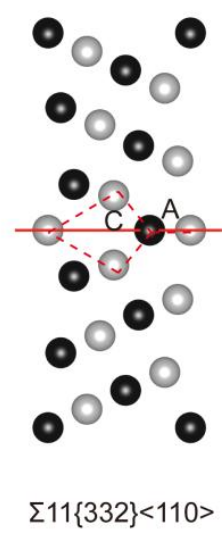

(a) $<110>$ STGBS

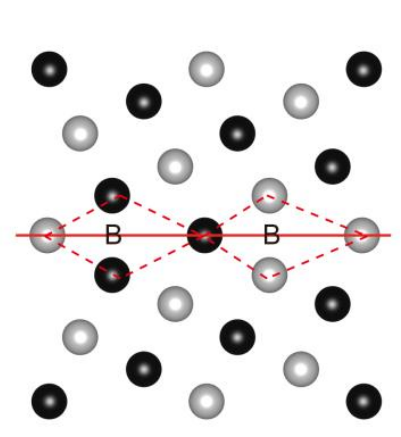

$\Sigma 5\{310\}<100>$

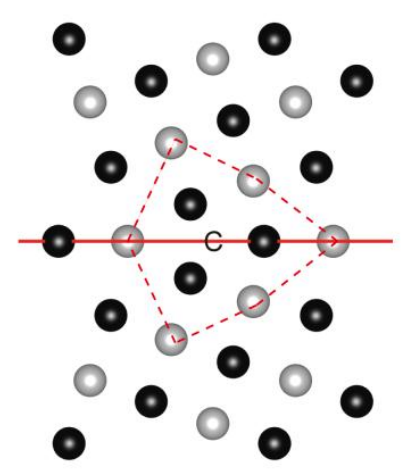

$\Sigma 5\{210\}<100>$

(b) $<100>$ STGBs

FIG. 4. The low- $\Sigma<110>$ and $<100>$ STGBs of bcc-Fe with the structural units being outlined. Black and gray balls denote atoms on different (a) $\{110\}$ planes and (b) $\{100\}$ planes. The different structural units are labeled as A, B, and C [18, 44]. 
So far, we reveal that, for a group of low $\Sigma$ GBs in bcc-Fe, the CSL and SU models may yield different structures on some GBs, and if so, they differ only by one vacancy on the CSL model GB. This shall be seen as a tentative hypothesis that demands further verification on high $\Sigma$ GBs in bcc-Fe and even on other crystal systems as well.

\section{B Vacancy formation at GBs}

Vacancy formation at GBs can be simulated by simply removing one atom from the GB region, right on the GB interface layer or from a sub-interface layer. The formation energy of a single GB vacancy, $E_{f}^{v a c}$, can be calculated as

$$
E_{f}^{v a c}=\frac{1}{2}\left(E_{G B}^{V}+2 \mu^{0}-E_{G B}^{0}\right),
$$

where $E_{G B}^{V}$ is the total energy of a fully relaxed GB containing one vacancy, $E_{G B}^{0}$ is the total energy of the corresponding perfect GB, and $\mu^{0}$ is the chemical potential of iron in its bcc bulk. In this section, vacancy formation energy will be evaluated as a function of the local atomic structure and also the distance from the GB interface plane. Meanwhile, by following the experimental conventions, we also define the GB vacancy density, $\rho_{\mathrm{V}}$, as

$$
\rho_{V}=n_{V} / \mathrm{A}
$$

where $n_{V}$ is the number of vacancies and A is the GB area.

Table II summarizes our calculation results for the two typical bcc-Fe GBs, i.e. the $\Sigma 5(310)[001]$ and the $\Sigma 5(210)[001]$ STGBs based on both the SU and CSL models, in comparison with other available DFT calculations in literatures. The comparison is made in terms of the total number of atoms in the supercells $(\mathrm{N})$, the corresponding vacancy density $\left(\rho_{V}\right)$, and the vacancy formation energies both on different GB layers $\left(E_{f}^{v a c}\right)$ and in bulk $\left(E_{B u l k}^{V}\right)$. Here L0 indicates the GB interface plane, L1 is the first sub-interface layer, and the other layers are named by analogy. In Table II, the GB vacancy formation energies are also compared to that in bulk. To calculate the vacancy formation energy in bulk, $E_{\text {bulk }}^{V}$, a $3 \times 3 \times 3$ (54-atom) supercell with a $9 \times 9 \times 9 \mathrm{k}$-mesh was employed to ensure the energy convergence within an error of $1 \mathrm{meV} /$ atom. 
Table II. Calculation results for vacancy formation on the SU-model $\Sigma 5(210)[001]$ and $\Sigma 5(310)[001]$ STGBs, in comparison with all relevant results in literatures.

\begin{tabular}{|c|c|c|c|c|c|c|c|c|c|}
\hline \multirow{2}{*}{$\begin{array}{c}\text { Grain } \\
\text { boundary }\end{array}$} & \multirow{2}{*}{ model } & \multirow{2}{*}{$\mathbf{N}$} & \multirow{2}{*}{$\underset{\left(\mathbf{n m}^{-2}\right)}{\rho_{V}}$} & \multicolumn{4}{|c|}{$E_{f}^{v a c}(\mathrm{eV})$} & \multicolumn{2}{|c|}{$E_{\text {Bulk }}^{V}(\mathrm{eV})$} \\
\hline & & & & Lo & L1 & $\mathbf{L 2}$ & L3 & $\begin{array}{c}\text { this } \\
\text { work }\end{array}$ & others \\
\hline \multirow{3}{*}{$\Sigma 5(210)[001]$} & SU & 76 & 2.77 & 0.89 & 1.07 & 1.26 & 2.09 & \multirow{7}{*}{2.11} & \\
\hline & CSL & 80 & 2.77 & 1.96 & 0.06 & 1.84 & 1.57 & & $2.07[45]$ \\
\hline & CSL & 40 & 5.55 & 1.62 & -0.55 & 0.82 & 1.31 & & $1.95[46]$ \\
\hline \multirow{2}{*}{$5(310)[001]$} & SU/CSL & 40 & 3.92 & 1.68 & 0.52 & 1.27 & 1.43 & & $2.00[47]$ \\
\hline & SU/CSL & 80 & 1.96 & 2.04 & 0.90 & 1.82 & 1.82 & & $\begin{array}{l}2.12[28] \\
2.13[48]\end{array}$ \\
\hline Ref. [22] & SU/CSL & 240 & 1.29 & 2.00 & 0.88 & 1.75 & 1.75 & & \\
\hline Ref. [28] & SU/CSL & 44 & 2.04 & 2.01 & 1.43 & 1.89 & 2.20 & & \\
\hline
\end{tabular}

As seen in Table II, all CSL-model results point to one major finding that, for either the $\Sigma 5(210)[001]$ or the $\Sigma 5(310)[001]$ STGB, the vacancy formation on the L1 layer requires the lowest cost in energy, no matter the supercell size and the GB vacancy density. This finding has been first noticed for $\Sigma 5(310)[001]$ by Zhou et al [28] and was referred as "the 1st nearest-neighbor (1NN) atom effect". Besides that, the vacancy formation energies for the L2 layers are also lower than those for the L0 layer of the $\Sigma 5(310)$ [001], as found in previous molecular dynamics (MD) simulations $[49,50]$. One might thus deduce that the GB vacancies are most likely found not right on the GB interface plane, but on the neighboring inner layers, which however is not true for the SU-modeled $\Sigma 5(210)[001]$. The reason for this will be discussed later in this section.

Also, as illustrated in Table II, we predict the bulk vacancy formation energy as $2.11 \mathrm{eV}$, comparable to the literature values of 1.95 2.13 eV. All these values are much higher than the vacancy formation on those near-GB layers, suggesting a strong tendency for bulk vacancies to segregate to GBs. As for the negative vacancy formation energy in the L1 layer for the CSL-modeled $\Sigma 5(310) \mathrm{GB}$, it does not necessarily suggest that forming a vacancy in the L1 layer is more favorable. Instead, we regard it as an evidence that the CSL model (as shown in Figure 3(c)) is not energetically stable, and is not appropriate to describe the $\Sigma 5(210)[001]$. 
To better understand the variation of vacancy formation energies among different GB layers, we thus employ the SU-model and analyze the calculated vacancy formation energy $\left(E_{f}^{v a c}\right)$ in three components: the atom removal energy $E_{G B}^{\text {remove }}$, the local relaxation energy $E_{G B}^{\text {local-R }}$, and the non-local lattice relaxation energy $E_{G B}^{\text {nonlocal }-R}$. They jointly constitute the vacancy formation energy, and each can be evaluated as follows.

$$
\begin{gathered}
E_{G B}^{\text {remove }}=\frac{1}{2}\left[E_{G B}^{v a c}(\text { unrelaxed })-E_{G B}^{0}+2 \mu^{0}\right] \\
E_{G B}^{\text {local }-R}=\frac{1}{2}\left[E_{G B}^{v a c}(\text { local_relaxed })-E_{G B}^{v a c}(\text { unrelaxed })\right] \\
E_{G B}^{\text {nonlocal }-R}=\frac{1}{2}\left[E_{G B}^{v a c}(\text { full_relaxed })-E_{G B}^{v a c}(\text { local_relaxed })\right] \\
E_{f}^{v a c}(\Sigma)=E_{G B}^{\text {remove }}+E_{G B}^{\text {local }-R}+E_{G B}^{\text {nonlocal }-R}
\end{gathered}
$$

Here, $E_{G B}^{v a c}$ (unrelaxed) is the static formation energy of a GB vacancy without any relaxation. $E_{G B}^{v a c}($ local_relaxed $)$ is the formation energy of a GB vacancy by allowing local relaxation only, i.e. all atoms inside the GB supercell are relaxed but the supercell size and shape are fixed. $E_{G B}^{\text {vac }}$ (full_relaxed) is the formation energy of a GB vacancy by allowing full relaxation, where all atoms inside the GB supercell and the supercell size and shape are all allowed to fully relax. The results are collected and compared with the bulk vacancy formation $\left(E_{\mathrm{Bulk}}^{V}\right)$ in Fig. 5.

As seen in Fig. 5, there is an evident competition between atom removal and lattice relaxation in forming a GB vacancy: the former consumes energy and the latter does the compensation. The energy gain from the local lattice relaxation has about the same order of magnitude as the energy cost for rigidly removing an atom. The impact of non-local relaxation is generally small but is not negligible. It is also suggested by Fig. 5 that, by comparing $E_{f}^{v a c}$ and $E_{\text {Bulk }}^{V}$, there shall be a strong tendency for bulk vacancies to segregate to GBs. The driving force is estimated to about $1 \sim 2 \mathrm{eV}$ per vacancy, depending on the specific structure of the targeted GB.

Due to the structural difference between a GB and the bulk, the vacancy formation energies at different GB layers normally increase inwards and approach gradually to its bulk value, as observed in Fig. 5(a) for the SU-model $\Sigma 5(210)[001]$. However, for all the CSL-model structures of $\Sigma 5(310)[001]$ and $\Sigma 5(210)[001]$ in Table II, the lowest vacancy formation energy is always found on the L1 layer. With carefully checking the CSL-model structures in Fig. 2, one shall notice that the atoms on the L1 layer are most closely-packed. 
Removing an atom from the L1 layer can be thus the most energy-favored, where maximal local relaxation can be expected to compensate the required removal energy. This is the case for the SU-model $\Sigma 5(310)[001]$ in Fig. 5(b) which happens to duplicate the structure of the CSL-model $\Sigma 5(310)[001]$. Moreover, this would be particularly true for the case of CSL-model $55(210)$ [001] where two L1-atoms form a very close pair (1-1') across the GB as seen in Fig. 2(b). Such a close proximity of atom pairs is absent in the SU-model $\Sigma 5(210)[001]$, and thus the inwards increasing trend of vacancy formation energy is sustained as seen in Fig. $5(\mathrm{a})$.

(a) $\Sigma 5\{210\}<100>$

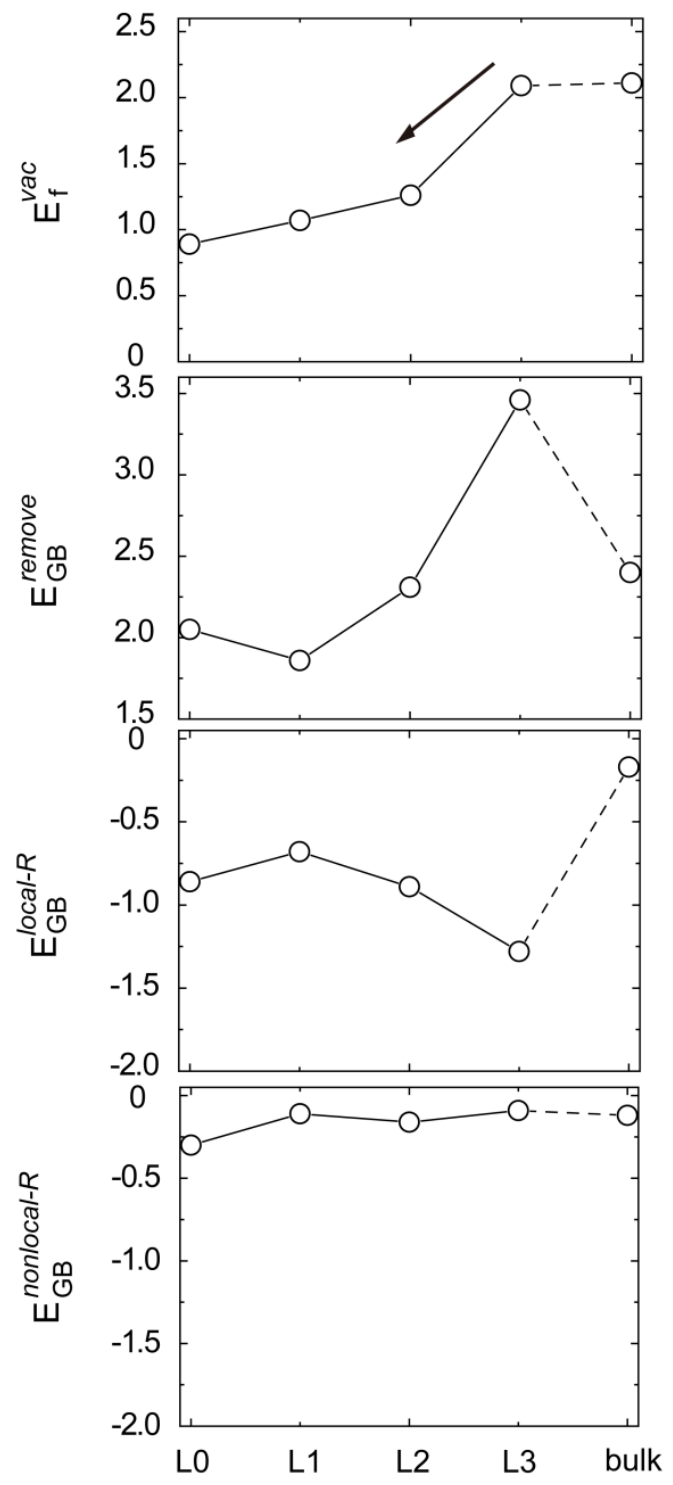

(b) $\Sigma 5\{310\}<100>$

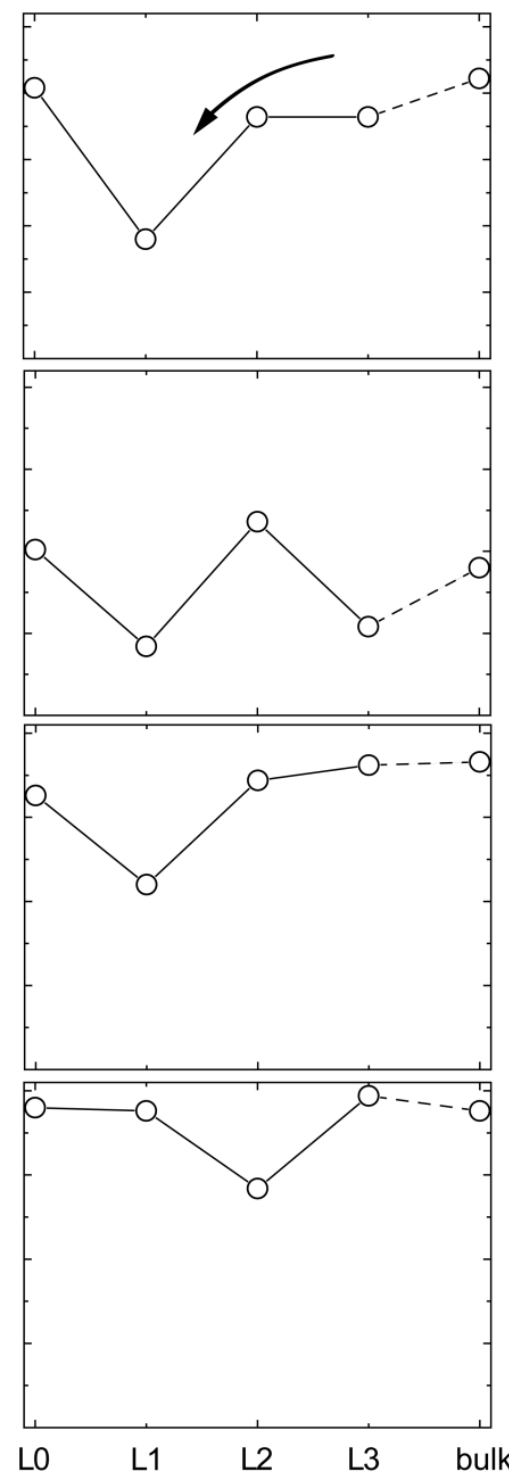

Location site of vacancy 
FIG. 5. The vacancy formation energies at different layers of (a) the SU-model $\Sigma 5(210)[001]$ and (b) the SU-model 55(310)[001] GBs. All energy units are in eV.

\section{CONCLUSIONS}

We have performed a systematic DFT based study on a group of representative low- $\Sigma(\Sigma \leq 11)$ symmetric tilt grain boundaries in bcc-Fe, using both the CSL and the SU models. The relation and applicability of the two GB models were evaluated, for the first time, for those low- $\Sigma$ bcc-Fe GBs. The following specific findings have been described.

(i) For the $\Sigma 3\{112\}<110>, \Sigma 3\{111\}<110>$, or $\Sigma 5\{310\}<100>$ GB, the CSL and SU models yield the same GB structure and thus the exactly same formation energy, while for the $\Sigma 5\{210\}<100>$, $\Sigma 9\{114\}<110>$, or $\Sigma 11\{332\}<110>$ GB, the two models yield different structures.

(ii) When the CSL and SU models yield different structures on some GBs, they differ only by one vacancy. For these cases, the SU model always predicts a much lower GB formation energy than the CSL does.

(iii) No matter which model was used, when compared to the DFT results, molecular dynamics (MD) methods always underestimate the GB formation energy, up to $50 \%$.

(iv) The SU model is suggested to be more appropriate than the CSL model for describing low- $\Sigma$ bcc-Fe GBs. The latter predicts a near-zero or even a negative formation energy of vacancy on the $\Sigma 5(210)[001]$

(v) Bulk vacancies have strong tendency to segregate to GBs in bcc-Fe. The driving force is estimated to be in the order of $1 \sim 2 \mathrm{eV}$ per vacancy, depending on the specific structure of the targeted GB. GB vacancy is energetically favorable to form on the first nearest neighbor layer of the $\Sigma 5(210)[001]$ and $\Sigma 5(310)[001]$ STGBs. 


\section{ACKNOWLEDGMENT}

This paper is based upon work supported by the National Science Foundation of China (No. 51471189). One of us (Y. Jiang) would like to thank the partial financial support from the National Basic Research Program of China (sub-contract No. 2014CB644001-2) and the Shenzhen Science and Technology Project (JCYJ20140509142357196). The computational resource at the High Performance Computing Center of Central South University is also gratefully acknowledged. 


\section{REFERENCES}

[1] S. J. Zinkle and J. T. Busby, Mater. Today 12, 12 (2009).

[2] E. E. Bloom, J. Nucl. Mater. 258, 7 (1998).

[3] M. J. Konstantinović, J. Nucl. Mater. 395, 75 (2009).

[4] Y. Wu, E. M. Haney, N. J. Cunningham, and G. R. Odette, Acta Mater. 60, 3456 (2012).

[5] T. Watanabe, Res Mechanica 11, 47 (1984).

[6] D. S. Lee, H. S. Ryoo, and S. K. Hwang, Mater. Sci. Eng. A 354, 106 (2003).

[7] J. P. Hirth, Metallurgical Transactions 3, 3047 (1972).

[8] A. Sutton and R. Balluffi, Oxford Langjahr PA, Wagner T, Lange FF, Ruhle M (2003) Epitaxial growth and structure of highly mismatched oxides with rock-salt structure on MgO. J Cryst Growth 256, 162 (1995).

[9] M. Kronberg and F. Wilson, AIME TRANS 185, 501 (1949).

[10] G. H. Bishop and B. Chalmers, Scripta Metallurgica 2, 133 (1968).

[11] G. Bishop and B. Chalmers, Philos. Mag. 24, 515 (1971).

[12] W. Read and W. Shockley, Phys. Rev. 78, 275 (1950).

[13] J. Li, Surf. Sci. 31, 12 (1972).

[14] K. Shih and J. Li, Surf. Sci. 50, 109 (1975).

[15] W. Bollmann, Crystal defects and crystalline interfaces (Springer Science \& Business Media, 2012).

[16] W.-Z. Zhang, and G. Weatherly, Acta Mater. 46, 1837 (1998).

[17] R. Balluffi, A. Brokman, and A. H. King, Acta Metallurgica 30, 1453 (1982).

[18] M. A. Tschopp, K. N. Solanki, F. Gao, X. Sun, M. A. Khaleel, and M. F. Horstemeyer, Phys. Rev. B 85, 064108 (2012).

[19] M. Müller, P. Erhart, and K. Albe, J. Phys.-Condes. Matter 19, 326220 (2007).

[20] Y. A. Du, L. Ismer, J. Rogal, T. Hickel, J. Neugebauer, and R. Drautz, Phys. Rev. B 84, 144121 (2011).

[21] N. Gao, C.-C. Fu, M. Samaras, R. Schäublin, M. Victoria, and W. Hoffelner, J. Nucl. Mater. 385, 262 (2009).

[22] L. Zhang, C.-C. Fu, and G.-H. Lu, Phys. Rev. B 87, 134107 (2013).

[23] E. Wachowicz, T. Ossowski, and A. Kiejna, Phys. Rev. B 81, 094104 (2010).

[24] Y. Zhang, W.-Q. Feng, Y.-L. Liu, G.-H. Lu, and T. Wang, Nuclear Instruments and Methods in Physics 
Research Section B: Beam Interactions with Materials and Atoms 267, 3200 (2009).

[25] L. Zhang, Y. Zhang, and G.-H. Lu, J. Phys.-Condes. Matter 25, 095001 (2013).

[26] M. Čák, M. Šob, and J. Hafner, Phys. Rev. B 78, 054418 (2008).

[27] S. K. Bhattacharya, S. Tanaka, Y. Shiihara, and M. Kohyama, J. Phys.-Condes. Matter 25, 135004 (2013).

[28] H.-B. Zhou, Y.-L. Liu, C. Duan, S. Jin, Y. Zhang, F. Gao, X. Shu, and G.-H. Lu, J. Appl. Phys. 109, $113512(2011)$.

[29] A. Suzuki and Y. Mishin, Interface Sci. 11, 425 (2003).

[30] G. Kresse and J. Furthmüller, Phys. Rev. B 54, 11169 (1996).

[31] P. E. Blöchl, Phys. Rev. B 50, 17953 (1994).

[32] G. Kresse and D. Joubert, Phys. Rev. B 59, 1758 (1999).

[33] J. P. Perdew, K. Burke, and M. Ernzerhof, Phys. Rev. Lett. 77, 3865 (1996).

[34] R. Kohlhaas, P. Dunner, and P. Schmitz, Z Angew Physik 23, (1967).

[35] C. Kittel, Introduction to solid state physics (Wiley, 2005).

[36] T. C. Leung, C. T. Chan, and B. N. Harmon, Phys. Rev. B 44, 2923 (1991).

[37] D. J. Singh, W. E. Pickett, and H. Krakauer, Phys. Rev. B 43, 11628 (1991).

[38] J.-H. Cho and M. Scheffler, Phys. Rev. B 53, 10685 (1996).

[39] M. Yamaguchi, M. Shiga, and H. Kaburaki, Mater. Trans. 47, 2682 (2006).

[40] M. A. Tschopp, F. Gao, L. Yang, and K. Solanki, J. Appl. Phys. 115, 033503 (2014).

[41] H. Momida, Y. Asari, Y. Nakamura, Y. Tateyama, and T. Ohno, Phys. Rev. B 88, 144107 (2013).

[42] H. Nakashima and M. Takeuchi, Tetsu-to-Hagane(Journal of the Iron and Steel Institute of Japan)(Japan) $86,357(2000)$.

[43] H. J. Monkhorst and J. D. Pack, Phys. Rev. B 13, 5188 (1976).

[44] O. Kapikranian, H. Zapolsky, C. Domain, R. Patte, C. Pareige, B. Radiguet, and P. Pareige, Phys. Rev. B 89, 014111 (2014).

[45] C.-C. Fu, F. Willaime, and P. Ordejón, Phys. Rev. Lett. 92, 175503 (2004).

[46] C. L. Fu, M. Krčmar, G. S. Painter, and X.-Q. Chen, Phys. Rev. Lett. 99, 225502 (2007).

[47] Y. Tateyama and T. Ohno, Phys. Rev. B 67, 174105 (2003).

[48] Y. Jiang, J. R. Smith, and G. R. Odette, Phys. Rev. B 79, 064103 (2009). 
[49] Y.-N. Wen, Y. Zhang, J.-M. Zhang, and K.-W. Xu, Comput. Mater. Sci. 50, 2087 (2011).

[50] T. Kwok, P. S. Ho, and S. Yip, Phys. Rev. B 29, 5354 (1984). 